\title{
What impacts the impact of rare events
}

\author{
Ido Erev • Ira Glozman • Ralph Hertwig
}

Published online: 26 February 2008

(C) Springer Science + Business Media, LLC 2008

\begin{abstract}
Kahneman and Tversky (Econometrica 47:263-291, 1979) argued that "unlikely events are either neglected or overweighted," but left the task of identifying factors that determine which of these contradicting biases occur to future research. We present four studies designed to tackle this issue in the context of decisions from incomplete descriptions. Our findings suggest that the impact of unlikely events increases when they become more similar to their comparison stimuli, and when they are explicitly presented. Using these factors we reversed the findings in variants of classic gambles in the history of decision research, the Allais and the St. Petersburg gambles.
\end{abstract}

Keywords Allais Paradox - Cumulative prospect theory - Decision weights · Decisions from experience $\cdot$ Mere presentation $\cdot$ Context effect

\section{JEL Classification $\mathrm{D} 80 \cdot \mathrm{D} 81$}

In their analysis of decision making under risk Kahneman and Tversky (1979) asserted: "Because people are limited in their ability to comprehend and evaluate extreme probabilities, highly unlikely events are either neglected or overweighted, and the difference between high probability and certainty is either neglected or exaggerated." (p. 283). In Tversky and Kahneman (1992), they reiterated the view that the probability weighting function "is not well-behaved near the endpoints, and

\footnotetext{
*We are grateful to Valerie M. Chase and Laura Wiles for editing the manuscript. We also thank the Jewish Communities of Germany Research Fund for a grant to the first author (Technion V.P.R. fund), and the Swiss National Science Foundation for their support of the third author (Grants 100013-107741/1 and 100014-118283/1). Part of this research was conducted while Ido Erev was a Marvin Bower Fellow at the Harvard Business School.

I. Erev $(\bowtie) \cdot$ I. Glozman

The Max Wertheimer Minerva Center for Cognitive Studies,

Faculty of Industrial Engineering and Management, Technion-Israel Institute of Technology,

Haifa 32000, Israel

e-mail: erev@tx.technion.ac.il

R. Hertwig

Department of Psychology, University of Basel, Basel, Switzerland

e-mail: ralph.hertwig@unibas.ch
} 
very small probabilities can be either greatly overweighted or neglected altogether" (p. 303). There are at least three ways to react to this view. One is to criticize it because neglecting versus overweighting rare events covers a wide range of psychological impacts of rare events and thus leaves little ground on which these statements can be falsifiable. Another reaction is - and it seems fair to say this reaction has been the predominant one of both Tversky and Kahneman (1992) and numerous later investigations (see Wakker and Tversky 1993; Wu and Gonzalez 1999; Chateauneuf and Wakker 1999; Prelec 2000) — to focus on one deviation from optimal weighting. Indeed, the prevailing view is that overweighting of rare events is a robust bias in decisions under risk. A third response is to return to Kahneman and Tversky's (1979) somewhat puzzling conjecture of the co-existence of overweighting and neglect of rare events, and to specify and test factors that determine whether rare events are overweighted or underweighted (which is tantamount to relative neglect). This is the path that we pursue in this paper.

We begin with a review of investigations of decision making in environments in which people receive incomplete descriptions of the payoff distributions. As we show, findings regarding the impact of rare events are mixed, with some studies suggesting overweighting and others underweighting. We next analyze how the investigations differ and focus on two well-known psychological factors that are likely to be responsible for the concurrence of both weighting patterns. The first factor is a context effect (see Garner 1954): Specifically, the implied subjective value of a target prospect is shifted toward the typical value of the prospect's alternatives (the comparison stimuli). The second factor is the mere-presentation effect (see a related concept in Hasher, Goldstein, and Toppino 1977): Specifically, the mere presentation of an outcome increases its impact.

Subsequent to our analysis, we conduct four studies that examine the significance of both factors for the impact of rare events. Study 1 finds that certainty equivalents and, by extension, conclusions regarding the weighting of events derived from them are highly sensitive to the distribution of the comparison stimuli. Study 2 shows that the mere-presentation effect can reverse the certainty effect (Allais paradox). Combining the impact of both facts, Studies 3 and 4 show how behavior in the St. Petersburg gamble can be altered and even reversed. Taken together, respondents in our studies exhibited behavior consistent with overweighting as well as neglect of rare events. Behavior consistent with overweighting was observed when rare events were presented, and comparison stimuli were similar to them. In contrast, leaving the rare event unmentioned and rendering the context dissimilar triggered behavior consistent with underweighting of rare events. We conclude with a discussion of the current results' implications for descriptive models of choice, and demonstrate that key results can be reproduced with a simple model. It assumes reliance on small samples of experiences and biases (during the sampling) toward experiences that are similar to the presented outcomes and the comparison stimuli.

\section{Previous studies of decision making from incomplete descriptions}

The current analysis distinguishes between two mutually exclusive classes of decision environments. The first class involves decisions under risk. In these 丝 Springer 
environments people receive complete descriptions of the payoff distributions of the available prospects. The monetary gambles examined by Kahneman and Tversky (1979) and in most follow up studies belong to this class. Hertwig, Barron, Weber, and Erev (2004) referred to such decisions as decisions from description. The second class of decision environments with which we will be concerned is decisions from incomplete descriptions. Here people have to rely on their existing knowledge and, if possible, collect new data. This class is more encompassing than that of decisions under uncertainty, as defined by Luce and Raiffa (1957), in which people know the possible outcomes, but do not know (and cannot compute) the probabilities with accuracy.

Decisions from incomplete description include problems with unknown outcomesfor example, the class of decisions from experience studied by Hertwig et al. (2004). In a typical study of decisions from experience people are asked to select one of two decks. They are told that their payoff will be determined by a random selection of one of the cards in the chosen deck. The decision makers do not receive a description of the content of the two decks, but can sample them (with replacement) before making their decision. Another important instance of decisions from incomplete descriptions are situations in which there is no complete representation of all outcomes and/or probabilities, even though a person could generate such a complete representation. The St. Petersburg gamble (presented in detail below) is an ideal example of such a situation. In theory, knowledge of simple arithmetic and the laws of probability would allow accurate computation of the relevant probabilities. In reality, gamblers will reason on the basis of an incomplete description.

\subsection{Overweighting rare events and neglecting them}

Fox and Tversky (1998) presented results indicating overweighting of rare outcomes in decisions under uncertainty. In one study, they presented their participants with options whose outcomes depended on the future 1995 National Basketball Association (NBA) championship. Each option consisted of a chance of receiving $\$ 160$ if a particular team, division, or conference won. For example, one of the options was: "\$160 if the Chicago Bulls win the NBA title; \$0 otherwise." In order to infer respondents' choices, the experimenters employed the method of certainty equivalence. The logic of this method is that for a given uncertain option, there exists a certain option that a person would just as soon choose as the uncertain one. This certain option (e.g., a certain gain of \$20) represents the person's certainty equivalent for the uncertain one. The uncertain option (e.g., \$160 if the Chicago Bulls win the NBA title; $\$ 0$ otherwise) was incompletely described: Although the possible outcome was specified, its probability was left open.

People made two decisions. First, they were asked to select between an uncertain option of the type "\$160 if Y [Chicago Bulls] wins the NBA title; \$0 otherwise" and nine certain payoffs uniformly distributed between $\$ 0$ and $\$ 160$ (i.e., $\$ 0, \$ 20, \$ 40, \ldots$, $\$ 160)$. Second, after making their initial judgment, participants went on to fine-tune their evaluations. Specifically, they were presented with nine sure payoffs that were uniformly distributed between the maximal rejected payoff and the minimal accepted payoff and asked to select the certain option that they would just as soon choose as the uncertain one. For example, if a participant preferred a given uncertain option to a sure $\$ 20$, but preferred a sure $\$ 40$ to the uncertain option, the payoffs for that participant 
were uniformly distributed between $\$ 22$ and $\$ 38$. Fox and Tversky (1998) constructed 14 uncertain options that offered to pay $\$ 160$ if a particular team (e.g., Chicago Bulls), division (e.g., Central), or conference (e.g., Eastern) won the championship. The study was conducted during the NBA playoffs, with eight teams remaining in the tournament. The eight teams represented four divisions and two conferences.

By comparing the median certainty equivalents for teams with those for divisions, Fox and Tversky (1998) found that the certainty equivalent for each division was smaller than the sum of all the certainty equivalents for the individual teams composing the division. For instance, the median certainty equivalents for the Chicago and Indiana teams were $\$ 49$ and $\$ 29$, respectively. The certainty equivalent for the respective division (Central), however, was only $\$ 49$. All else being equal, a given team's chance of winning the NBA championship was .125 , and a given division's chance of including the winning team was .25. Thus, comparing the certainty equivalents for the individual teams to those for the division indicated that people had overweighted the event that a particular team would win, relative to the more likely event that the winner would come from a particular division. According to Fox and Tversky, the oversensitivity to rare outcomes can be attributed to two (nonexclusive) causes. The first, consistent with support theory (Tversky and Koehler 1994), is that people tend to overestimate small probabilities when explicitly asked to estimate the probability of an event (e.g., a given team will win the NBA championship). The second cause, posited by cumulative prospect theory, is that the (estimated) small probabilities receive too much weight.

Are we doomed to pay undue attention to rare events, as the above theory and results suggest? A very recent set of studies reveals quite different behavior. Hertwig et al.'s (2004) study is representative of these investigations and their conclusions. Here, two groups of participants saw six decision problems. The two groups differed only with respect to how they learned about the options' outcomes and likelihoods. In the description group, options were described as follows:

get 4 with probability .8; 0 otherwise, or get 3 for sure.

Thus, respondents made decisions from descriptions, as did participants in Kahneman and Tversky's (1979) studies and in many other studies on human risky choice. ${ }^{1}$ In the experience group, the information describing the options was not conveniently displayed. Instead, respondents saw two buttons on the computer screen and were told that each button was associated with a payoff distribution. Clicking on a given button elicited the sampling of an outcome (with replacement) from its distribution. In the above problem, for example, drawing from one distribution led to the outcome " 4 " in $80 \%$ of all draws and to the outcome " 0 " in $20 \%$ of all draws. Sampling from the other distribution always resulted in the outcome " 3 ". Respondents could sample however often they wished. By repeatedly experiencing the contingency between choices and outcomes, participants could gradually acquire knowledge about the options' payoff structure. Once they stopped

\footnotetext{
${ }^{1}$ In a recent meta-analysis of all studies involving decisions between a two-outcome risky prospect and a sure thing (with equal expected value), Weber, Shafir, and Blais (2004) found that all 226 choice situations called for decisions from descriptions.
} 
sampling, they indicated their preferred option, and, after completing all problems, respondents played out the selected options and received real monetary payoffs.

Choices in the experience and description groups differed drastically. This divergence is illustrated in Table 1, which shows the percentage of respondents who chose option $H$ (i.e., the option with the higher expected value) in the six problems that Hertwig et al. (2004) studied. For instance, in Problem 1, 88\% of respondents in the experience group selected $H$, whereas only $36 \%$ in the description group did so (similar to the $20 \%$ of participants who chose $H$ in Kahneman and Tversky's 1979 study). In order to clarify the difference between the two groups we divided the six problems using the premise that people treat rare and frequent events as if they are equally likely (which is tantamount to assuming that people extremely overweight rare events). Based on this premise, one can predict the choice of option $L$ (i.e., the option with the lower expected value) in the first two problems, and the choice of option $H$ in the last four problems. As Table 1 shows, the modal response in the description group deviates from maximization when the undue impact of rare events implies choice of option $L$. This pattern is consistent with overweighting of rare events. The opposite pattern is observed in the experience group.

Table 1 Summary of the proportion of $\mathrm{H}$ choices observed in Hertwig et al. (2004), inferred choice proportions in Study 1, and relevant predictions of the primed-sampler model

\begin{tabular}{|c|c|c|c|c|c|c|c|c|c|}
\hline \multirow{2}{*}{$\begin{array}{l}\text { Decision } \\
\text { Problem }\end{array}$} & \multicolumn{2}{|l|}{ Options $^{\mathrm{a}}$} & \multicolumn{2}{|c|}{ Hertwig et al. (2004) } & \multicolumn{2}{|c|}{ Study $1^{b}$} & \multicolumn{3}{|c|}{ Primed-sampler model $^{\mathrm{c}}$} \\
\hline & $\mathrm{H}$ & $\mathrm{L}$ & Description & Experience & $\begin{array}{l}\text { AS- } \\
\text { Rare }\end{array}$ & $\begin{array}{l}\text { AS- } \\
\text { Likely }\end{array}$ & Experience & $\begin{array}{l}\text { AS- } \\
\text { Rare }\end{array}$ & $\begin{array}{l}\text { AS- } \\
\text { Likely }\end{array}$ \\
\hline 1 & $4,0.8$ & 3 & 36 & 88 & 44 & $78^{*}$ & 74 & 48 & 87 \\
\hline 2 & -3 & $-4,0.8$ & 28 & 56 & 34 & $85^{*}$ & 74 & 48 & 87 \\
\hline \multicolumn{3}{|c|}{$\begin{array}{l}\mathrm{P}(\mathrm{H}) \text { when undue impact of rare } \\
\text { events implies } L \text { choice }\end{array}$} & 32 & 72 & 39 & $82 *$ & 74 & 48 & 87 \\
\hline 3 & $32,0.1$ & 3 & 48 & 20 & 81 & $11 *$ & 41 & 78 & 31 \\
\hline 4 & $4,0.2$ & $3,0.25$ & 64 & 44 & 65 & 56 & 54 & 59 & 64 \\
\hline 5 & $32,0.025$ & $3,0.25$ & 62 & 12 & 67 & 52 & 22 & 73 & 49 \\
\hline 6 & -3 & $-32,0.1$ & 64 & 28 & 65 & $37 *$ & 41 & 78 & 31 \\
\hline \multicolumn{3}{|c|}{$\begin{array}{l}\mathrm{P}(\mathrm{H}) \text { when undue impact of rare } \\
\text { events implies } H \text { choice }\end{array}$} & 59 & 26 & 69 & $39 *$ & 39 & 72 & 44 \\
\hline
\end{tabular}

Italicized values indicate the options including rare events.

$H=$ option with the higher expected value; $L=$ option with the lower expected value.

${ }^{a}$ For each option, only one outcome is given, followed by its probability; the second outcome, which is not stated, was 0 and occurred with a probability complementary to the stated one. For instance, the outcomes of the $H$ option in Problem 1 were 4 with a probability of .8 and 0 with a probability of .2 ; the outcome of the $L$ option was 3 with a probability of 1.0 .

${ }^{\mathrm{b}}$ Percentages of participants choosing the $H$ option, as derived from the certainty equivalents observed in the AS-Rare and AS-Likely context conditions, respectively. AS in the condition name stands for "Alternatives Similar to". In the AS-Rare condition the alternatives are similar to the rare outcome: the midpoint of the range of certain payoffs is between the gambles' expected value and the least likely alternative. In the AS-Likely condition, the midpoint of the range of certain payoffs is between the gambles' expected value and the most likely alternative.

${ }^{\mathrm{c}}$ The percentage of participants choosing the $H$ option, as predicted by the primed-sampler model.

* Indicate that these percentages are significantly different from the AS-Rare context condition $(p<0.05)$. 


\subsection{The impact of context and mere presentation}

The mixed findings presented above can be explained as a product of two wellknown effects in human perception and cognition, the context and the merepresentation effects, respectively.

\subsubsection{Context matters}

The context effect represents the frequently made observation that judgments and choices are markedly influenced by the context in which they are made, specifically by the distribution of comparison stimuli. For illustration, consider a classic study in psychophysics. Garner (1954) asked respondents to find a tone that would seem "half as loud" as a target tone. People were asked in a choice-based procedure to determine whether each comparison tone was more or less than half as loud as the target tone. By manipulating the distributions of comparison stimuli (i.e., the context), he found that the tone inferred to be "half as loud" was in fact the median of the comparison stimuli.

As demonstrated by Birnbaum (1992, and see related observations in Gregory, Lichtenstein, and Slovic 1993; Harbaugh, Krause, and Vesterlund 2004; Stewart, Chater and Brown 2006), the certainty equivalence method used by Fox and Tversky (1998) is analogous to Garner's (1954) procedure. As a consequence the process of estimating certainty equivalents is also influenced by the context, that is, the comparison stimuli. ${ }^{2}$ The context effect implies a regression toward the midpoint of the response scale (see Erev, Wallsten, and Budescu 1994 for related observations). Depending on the chosen context, regression, in turn, can yield choices consistent with the notion of the undue impact of rare events. Let us, for example, assume that the gamble is "\$160 if team $X$ wins; $\$ 0$ otherwise," and that its true value is $\$ 10$. If the gamble is compared with certain gains evenly distributed between $\$ 0$ and $\$ 160$ (with $\$ 80$ being the midpoint of the series of certain gains), then-assuming regression toward the scale's midpoint - the estimated true value will fall between $\$ 10$ and $\$ 80$. Values in this range would be consistent with the interpretation of rare events having undue impact.

One way to interpret the context effect is that the impact (attention, weighting) of a particular outcome increases with its "relative similarity" to the comparison stimuli (the listed alternatives that make up the context). Relative similarity is abstracted here with reference to the deviation from the prospect's expected value. A comparison stimulus is assumed to be most similar to that outcome deviating from the prospect's expected value in the same direction as the stimulus. For example, since the expected value of the gambles considered by Fox and Tversky (1998) is $\$ 20$ (on average), the comparison context implies that the rare outcome $(\$ 160)$ is more similar to most stimuli than the likely outcome $(\$ 0)$.

\footnotetext{
${ }^{2}$ This observation does not imply that studies that use the certainty equivalence lead to biased results. Rather, it implies that the observed results (the implied deviation from optimal weighting of rare events) can be safely generalized to similar contexts, but not to any other contexts.
} 


\subsubsection{Mere presentation matters}

Another simple factor that is likely to contribute to the impact of a rare event is whether or not it is explicitly presented. Unlike in Hertwig et al. (2004), respondents in Fox and Tversky's (1998) study were explicitly presented with the rare outcomes, that is, they were asked to consider the chances that specific, explicitly stated teams would win the NBA championship. It is often said that to name something is to own it. By analogy, we propose that to present a rare event is to increase its psychological impact on the decision-making process. We refer to this as the mere-presentation effect. It belongs to a set of phenomena in which mere presentation of an event (even in the absence of information about its likelihood) alters a wide range of responses.

For instance, even in the absence of confirming evidence and inferences, considering a proposition appears to enhance its subjective truth (Fiedler 2000; Fiedler et al. 1996; Erev, Shimonowitch, Schurr, and Hertwig 2008). Similarly, Gregory, Cialdini, and Carpenter (1982) demonstrated that having participants imagine the occurrence of events (e.g., winning a free trip, being arrested) caused the estimated likelihood of those events to increase. Hasher, Goldstein, and Toppino (1977) observed that confidence in the truth of an assertion increases after the assertion has been presented repeatedly, independent of its truth or falsity (see also Hertwig, Gigerenzer, and Hoffrage 1997). Moreover, listing the outcomes of an option affects its attractiveness. For instance, Birnbaum and Martin (2003) demonstrated that increasing the ratio between the number of good and bad outcomes that are explicitly listed for an option makes the option more attractive. Returning to Fox and Tversky's (1998) study, we suggest that the mere presentation of specific teams contributed to certainty equivalents that are consistent with the interpretation that respondents overweighted the odds of a specific individual team (rare event) winning the NBA championship (compared with, for instance, the division's certainty equivalents).

\section{Experimental studies}

Our thesis is that two well-known psychological effects, context and mere presentation, can contribute to the difference between studies that document overweighting and relative neglect of rare events in decisions from incomplete descriptions. Can we use these effects to reverse the direction of the implied deviation from optimal weighting of rare events? We conducted four experiments to investigate this possibility.

\subsection{Study 1. Does context affect certainty equivalents?}

Study 1 tested the thesis that the impact of rare events, inferred from certainty equivalents, is determined by their comparison stimuli. To this end, we combined the experimental method in Hertwig et al.'s (2004) sampling paradigm with the certainty equivalence method used by Fox and Tversky (1998). 


\subsubsection{Method}

Participants (in the main part of the study) were 75 students enrolled at the Technion Institute of Technology, Haifa, Israel. Most of them were second- or third-year students in industrial engineering or economics who had taken at least one probability course and one economics course. In addition to payoffs contingent on their choices, participants received 20 shekels (about $\$ 4.50$ U.S.) for showing up. The performance-contingent payoffs ranged between 10 and 28 shekels (between about \$2 U.S. and about \$6 U.S.).

Respondents were asked to compare one of seven two-outcome gambles investigated by Hertwig et al. (2004) to a context of certain payoffs (see Tables 1 and 2). Before they made the comparisons, participants first saw a deck of cards on the computer screen. The instructions stated that the deck consisted of two types of events (i.e., red and blue cards), from which respondents were encouraged to sample thus finding out the proportion of red and blue cards. The proportions of red cards in each deck corresponded to the probabilities in one of the seven gambles. For example, the gamble "32 with probability $0.1 ; 0$ otherwise" corresponded to a deck with $10 \%$ red cards, and $90 \%$ blue cards. Once participants stopped sampling, they were presented with a gamble-for instance, "Win 32 if the card is red; 0 otherwise" - and asked to choose between this gamble and various certain payoffs.

Each participant was randomly assigned to one of two conditions, which differed with regard to the context, that is, the range of certain payoffs (comparison stimuli) to which the uncertain payoff was compared. In the first context condition $(n=47)$ the certainty equivalence method was similar to that used by Fox and Tversky (1998). Specifically, the range of certain payoffs was defined by the lowest payoff and highest payoff that could be obtained in the gamble under consideration (see Table 2). The choice between the gamble and the comparison stimuli proceeded in two steps. The first required comparing the gamble to nine certain payoffs, spaced uniformly between the lowest and the highest payoffs. For example, the gamble "32 with probability .1; 0 otherwise" offered 32 points if the selected card was red and 0 points if the selected card was blue. As Fig. 1 illustrates, this gamble's certain payoffs were spaced evenly between 0 (lowest payoff) and 32 (highest payoff). The midpoint of the range of certain payoffs was thus located between the gamble's expected value (here 3.2) and its rare outcome (here 32). Consequently, most comparison alternatives were more similar (i.e., to the same side of the expected value) to the rare outcome (32) than to the likely outcome (0). We refer to this condition as the "Alternatives Similar to Rare" (AS-Rare) context condition.

The second step in the AS-Rare context required comparing the gamble to a new context of certain payoffs. The payoffs ranged between the maximum certain payoff that a respondent initially rejected and the minimum certain payoff he or she accepted. If, for example, a participant preferred the gamble "32 with probability .1; 0 otherwise" to the certain payoff of 16 but preferred the sure payoff of 20 over the gamble, then the sure payoffs in the second round would be uniformly distributed between 16 and 20 respectively (Fig. 1). The outcome of the second round determined the participant's estimated certainty equivalent (CE) value: It was the average of the highest rejected sure payoff and the lowest accepted sure payoff. Participants were told that their choices should be consistent. When a violation of dominance (such as 


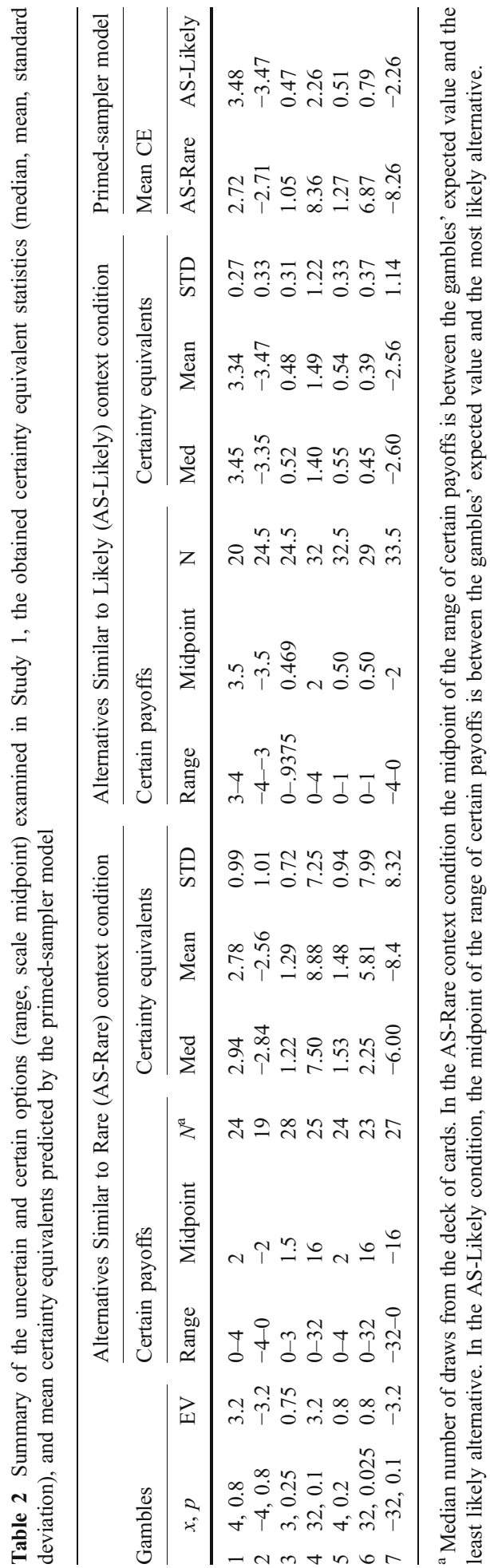




\begin{tabular}{|c|c|c|c|c|c|c|c|}
\hline 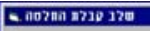 & & & निवर & 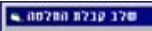 & & & Enx \\
\hline $\begin{array}{l}32 \text { points if } X \text { red } \\
0 \text { points if } X \text { blue }\end{array}$ & - & 8 points for sure & $r$ & $\begin{array}{l}32 \text { points if } X \text { red } \\
0 \text { points if } X \text { blue }\end{array}$ & $c$ & 16 points for sure & $c$ \\
\hline $\begin{array}{l}32 \text { points if } \times \text { red } \\
0 \text { points if } X \text { blue }\end{array}$ & 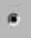 & 4 points for sure & $r$ & $\begin{array}{l}32 \text { points if } \times \text { red } \\
0 \text { points if } \times \text { blue }\end{array}$ & $c$ & 16.5 points for sure & $c$ \\
\hline $\begin{array}{l}32 \text { points if } X \text { red } \\
0 \text { points if } X \text { blue }\end{array}$ & 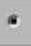 & 8 points for sure & r & $\begin{array}{l}32 \text { points if } X \text { red } \\
0 \text { points if } X \text { blue }\end{array}$ & $c$ & 17 points for sure & $c$ \\
\hline $\begin{array}{l}32 \text { points if } X \text { red } \\
0 \text { points if } X \text { blue }\end{array}$ & $\pi$ & 12 points for sure & c & $\begin{array}{l}32 \text { points if } X \text { red } \\
0 \text { points if } X \text { blue }\end{array}$ & $c$ & 17.5 points for sure & $c$ \\
\hline $\begin{array}{l}32 \text { points if } X \text { red } \\
0 \text { points if } X \text { blue }\end{array}$ & $\bullet$ & 16 points for sure & $r$ & $\begin{array}{l}32 \text { points if } X \text { red } \\
0 \text { points if } X \text { blue }\end{array}$ & $r$ & 18 points for sure & $c$ \\
\hline $\begin{array}{l}32 \text { points if } X \text { red } \\
0 \text { points if } X \text { blue }\end{array}$ & $c$ & ints for sure & & $\begin{array}{l}32 \text { points if } X \text { red } \\
0 \text { points if } X \text { blue }\end{array}$ & $c$ & 18.5 points for sure & $c$ \\
\hline $\begin{array}{l}32 \text { points if } X \text { red } \\
0 \text { points if } X \text { blue }\end{array}$ & $c$ & 24 points for sure & * & $\begin{array}{l}32 \text { points if } X \text { red } \\
0 \text { points if } X \text { blue }\end{array}$ & $r$ & 19 points for sure & $c$ \\
\hline $\begin{array}{l}32 \text { points if } X \text { red } \\
0 \text { points if } X \text { blue }\end{array}$ & $c$ & 28 points for sure & e & $\begin{array}{l}32 \text { points if } X \text { red } \\
0 \text { points if } X \text { blue }\end{array}$ & $r$ & 19.5 points for sure & $c$ \\
\hline $\begin{array}{l}32 \text { points if } X \text { red } \\
0 \text { points if } X \text { blue }\end{array}$ & $c$ & 32 points for sure & $r$ & $\begin{array}{l}32 \text { points it } X \text { red } \\
0 \text { points if } X \text { blue }\end{array}$ & $r$ & 20 points for sure & $c$ \\
\hline
\end{tabular}

Fig. 1 Example of the two rounds of choices performed in Study 1. In the first round (left panel), a hypothetical respondent prefers the gamble (assuming Problem 3) to a certain payoff of 16 points but prefers a certain payoff of 20 points to the gamble. As a consequence, the context of certain payoffs in the second round (right panel) ranges between 16 and 20

preferring a certain payoff of 0 to an uncertain payoff of 32 or 0 , respectively) or an internal inconsistency (preferring the gamble over the certain payoff $a$ while preferring the sure payoff $b$ over the uncertain prospect, when $b<a$ ) was detected, the respondent was instructed to make the choice again (as in Fox and Tversky 1998). Note that the range of uncertain payoffs in the first round of comparisons was determined by the gamble, whereas in the second round a respondent's first-round responses determined it.

The setup in the second condition $(n=27)$ was identical to the previous condition in all respects except one. Here, each gamble was compared with 11 values equally spaced between the option's most likely outcome (MLO) and a near expected value (NEV) outcome, where NEV is computed as follows: $\mathrm{NEV}=\mathrm{EV}+.25(\mathrm{EV}-\mathrm{MLO})$. For example, the gamble "32 with probability .1; 0 otherwise" was compared with certain payoffs equally spaced between 0 and $4[=3.2+.25(3.2-0)]$. In this condition, the midpoint of the range of certain payoffs was located between a gamble's expected value and its most likely outcome. Thus, most comparison alternatives were more similar to the likely outcome than to the rare outcome. We refer to this condition as the Alternatives Similar to Likely (AS-Likely) context condition.

\subsubsection{Results}

Table 2 displays the median number of draws (taken during the information sampling stage), and the certainty equivalent (CE) statistics obtained in both conditions. Although our respondents drew larger samples than those in Hertwig et al.'s (2004), the number of draws did not significantly differ between the both conditions (average medians of 24 and 28, respectively). The CE statistics, however, varied markedly across conditions. Across all 14 tests (seven problems times two conditions), the mean and median certainty equivalents deviated from the gambles' expected values in the direction of the midpoint of the range of certain payoffs. For 
example, in Problem 4 (32, .1; 0 otherwise; $E V=3.2)$, the median CE was 7.5 in the AS-Rare context condition (median comparison alternative $=16$ ), but 1.40 in the ASLikely context condition (median comparison alternative $=2$ ).

Three different analyses demonstrate that the two conditions give rise to discrepant conclusions regarding the impact of rare events. The first analysis compares the median CE values to the expected values of the gambles. ${ }^{3}$ In the ASRare context, the median CEs for all seven gambles deviate from the EVs in the direction of the rare outcomes. A sign test shows that this regularity is significant $(p<.02)$. In the AS-Likely context, in contrast, the median CEs for all seven gambles deviate from the EVs in the direction of the most likely outcomes. Again, a sign test shows that this regularity is significant $(p<.02)$.

The second analysis compares the current results with those observed by Hertwig et al. (2004). To make this comparison possible, we used the estimated CE values to infer choices in Hertwig et al.'s six decision problems. For example, if a person's certainty equivalent in option $H$ in Problem 1 was larger than the value 3 , we assumed that his or her choice would be the option with the higher expected value (i.e., the risky option " 4 with probability .8; 0 otherwise"). Table 1 displays these inferred choices. Consistent with the previous analysis, choices in the AS-Rare context are as if rare events have undue impact. Averaged across Problems 1 and 2, in which undue impact of the rare event (here " 0 ") reduces the attractiveness of option $H$, the implied proportion of $H$ choice is $39 \%$ and $82 \%$ in the AS-Rare and AS-Likely contexts, respectively $(t[73]=-5.3, p<.0001)$. Averaged across Problems $3,4,5$ and 6 , in which less than due impact of the rare event reduces the attractiveness of option $H$, the implied proportion of $H$ choice is $69 \%$ and $39 \%$ in both conditions, respectively $(t[73]=4.75, p<.0001)$. These results demonstrate that depending on the context of certain payoffs, choices emerge suggesting opposite conclusions about rare events' impact.

The final analysis focuses on Problems 1 and 4 only (see Table 1), variants of the Allais common ratio problems. Notice that Problem 4 is a linear transformation of Problem 1, and was created by dividing the probabilities of Problem 1's desirable outcomes (4 and 3) by four. According to expected utility theory, such a transformation should not affect people's preferences but, as Allais (1953) observed, it does, and this finding had a crucial impact on the development of descriptive models of risky choice. Kahneman and Tversky (1979) interpreted people's choices in both problems in terms of a certainty effect: That is, the elimination of chance of winning has more impact when it alters the character of the prospect from a sure gain to a probable one, compared to gambles in which both the original and the reduced prospects are uncertain. As a consequence, most people will choose the certain event, option $L$, in Problem 1 (" 4 with probability .8; 0 otherwise"), but prefer option $H$ in Problem 4. Indeed, Kahneman and Tversky reported $20 \%$ and $65 \%$ of participants, respectively, choosing option $H$ in both problems. Similarly, $44 \%$ and $65 \%$ of participants did so in the AS-Rare context (Table 1). In the AS-Likely context, in contrast, $78 \%$ and $56 \%$ of participants chose option $H$, thus amounting to a reversed certainty effect (see also Barron and Erev 2003; Hertwig et al. 2004). In

\footnotetext{
${ }^{3}$ Because the means are biased by the limited range in the AS-Rare-context, we focus on the medians.
} 
other words, by merely changing the range of comparison stimuli, the certainty effect can be reversed.

One difficulty in the interpretation of the previous results lies in the fact that the two conditions differ on three dimensions: the midpoint of the range of the uniformly distributed sure payoffs (one measure of the judgmental context), the width of the range, and the number of comparisons. In the AS-Rare context, participants were asked to consider a wide set of alternatives (see Table 2) and perform two sets of nine comparisons; in the AS-Likely context, the range was more restricted (see Table 2) and only one set of 11 comparisons was required. In order to examine the potential impact of these differences we conducted a third condition. This "OneRestricted-Rare" condition $(n=24)$ was a direct replication of the AS-Likely context (one set of 11 comparisons and restricted range) with one exception: The distance of the two extreme sure payoffs from the gamble's EV was reversed. One extreme payoff was set to equal NEV' $=\mathrm{EV}-.25(\mathrm{EV}-\mathrm{MLO})$. The second extreme payoff was placed on the other side of the EV and located towards the least likely outcome (TLLO). To achieve the same restricted range as in the AS-Likely context the exact value of this point was TLLO $=\mathrm{NEV}^{\prime}+(\mathrm{NEV}-\mathrm{MLO})$. For example, in Problem 4 $(32, .1 ; 0$ otherwise) the range was $(0,4)$ in the AS-Likely context (see Table 2; $\mathrm{MLO}=0, \mathrm{EV}=3.2$, and $\mathrm{NEV}=4)$. In the One-Restricted-Rare condition, NEV' was $2.4(3.2-.25(3.2-0))$ and TLLO was $6.4(2.4+(4-0))$, thus creating a width of the range 2.4 to 6.4 , that is identical to the AS-Likely context condition. The results reveal the pattern observed in the AS-Rare context. Specifically, the median CE values were closer to the rare outcome than the CE values observed in the AS-Likely context in all seven cases. In six of the seven cases the medians of the OneRestricted-Rare condition fell between the gamble's expected value and the rare outcome.

To conclude, Study 1 demonstrates that certainty equivalents are highly sensitive to the distribution of the comparison stimuli. As predicted by the context effect, choices suggested overweighting of rare events when most comparison stimuli were similar to (located to the same side of the EV) as the rare outcome, and underweighting when most comparison stimuli were similar to the more likely outcome.

2.2 Study 2. Can the mere-presentation effect reverse the certainty effect?

In Study 2, we turn to the mere-presentation effect and its impact of rare events. As in Study 1, we focused on a scenario in which respondents sample information from payoff distributions.

\subsubsection{Method}

Participants were 109 Technion students drawn from the same pool used in Study 1. They received 20 shekels (about $\$ 4.50$ U.S.) for showing up. In addition, they could earn up to 25 shekels (about \$5 U.S.), depending on their choices. The betweensubjects design involved two conditions. The procedure in the blank condition $(n=$ 59) was identical to that in Hertwig et al.'s (2004) experience group: Participants saw two blank buttons on the computer screen and were told that each button was 
associated with a payoff distribution. Clicking on a given button elicited the sampling of an outcome (with replacement) from its distribution. Respondents could sample in whatever order they desired, and how often they wished. Once they stopped sampling, they were asked to indicate their preferred option. The procedure in the mere-presentation condition $(n=50)$ was the same except for one critical difference: The possible outcomes in each distribution were explicitly stated on the buttons. For example, when the payoff distribution was " 4 with probability .8; 0 otherwise," the button said " 4 or $0 ., 4$

Table 3 displays the six decision problems to which participants responded. The problems included three variants of the common ratio problems, namely, the variant examined in Study 1 (Problems 1 and 4) and two others. We focused on Allais-like problems because they have offered the most influential demonstrations of nonlinear weighting of probabilities (see $\mathrm{Wu}$ and Gonzalez 1998). How did the mere presentation of rare events change their impact?

\subsubsection{Results}

Table 3 displays the median number of draws per decision problem (separated for risky and safe options), and the proportions of risky choices in the two conditions. First, the results show similar patterns of sampling. Across all problems and options, the average median sample sizes in the mere-presentation and blank conditions were 14.6 and 13.4, respectively. Choices in the two conditions, however, differed drastically. The mere-presentation condition yielded the original certainty effect for all three pairs of gambles: That is, the safe option was more attractive when it provided a positive payoff with certainty. The effect is significant in Pair $1(t[49]=$ $2.65, p<.02)$ and Pair $3(t[49]=3.31, p<.01)$, but not in Pair $2(t[49]=1.23, n s)$. The blank condition, in contrast, yielded a reversed certainty effect in Pair $1(t[57]=2.74$, $p<.01)$ and Pair $2(t[57]=2.01, p<.05)$, but not in Pair $3(t[57]=-1.48, n s)$. The latter finding may be related to the observation that a reversed certainty effect is less likely to occur when the option $R$ has a lower expected value than option $S$ (Ish Shalom 2004).

The above pattern of results is also corroborated by an analysis of the certainty effect itself (Table 3). The certainty effect is the difference in the percentages of risky choices per pair of decision problems. Averaged across all pairs, the certainty effect differs significantly between the conditions $(t[106]=4.05, p<.001)$. It also differs in two of the three pairs, namely, Pair $1(t[106]=2.26, p<.05)$ and Pair $2(t[106]=3.82$, $p<.005)$, but not in Pair $3(t[106]=1.35, n s)$. These results are consistent with the suggested impact of the mere-presentation effect. When rare events were explicitly presented, respondents made choices as if rare events had more impact than they deserved, relative to their objective probability. When rare events were not explicitly presented - as was the case in Hertwig et al.'s (2004) study - choices were made as if rare events had less impact than they deserved.

\footnotetext{
${ }^{4}$ As a consequence, participants in the mere-presentation condition had more information (they knew the outcomes). In our view this fact is not an experimental artifact. Rather we suspect that in many real-world domains (e.g., purchase of insurance) people are aware of the possible outcomes but hold little information about their probabilities. In Study 3 we consider an environment in which presentation of the possible outcomes does not add information.
} 
Table 3 Summary of the gambles presented in Study 2, the proportion of respondents selecting the risky options in the two experimental conditions, and the respective proportions predicted by the primed-sampler model

\begin{tabular}{|c|c|c|c|c|c|c|c|c|c|c|c|c|c|}
\hline \multicolumn{4}{|c|}{ Gambles } & \multicolumn{5}{|c|}{ Mere presentation $(n=50)^{\mathrm{a}}$} & \multicolumn{3}{|c|}{ Blank $(n=58)$} & \multicolumn{2}{|c|}{$\begin{array}{l}\text { Primed-sampler } \\
\text { Model: } \mathrm{P}(\mathrm{R})\end{array}$} \\
\hline Pairs & $\begin{array}{l}\text { Decision } \\
\text { Problems }\end{array}$ & $\mathrm{R}$ & $\mathrm{S}$ & $\begin{array}{l}\mathrm{N} \\
(\mathrm{R})^{\mathrm{a}}\end{array}$ & $\begin{array}{l}N \\
(S)^{a}\end{array}$ & $\begin{array}{l}\mathrm{P} \\
(\mathrm{R})^{\mathrm{b}}\end{array}$ & $\begin{array}{l}\text { Certainty } \\
\text { effect }^{c}\end{array}$ & $\begin{array}{l}\mathrm{N} \\
(\mathrm{R})\end{array}$ & $\begin{array}{l}\mathrm{N} \\
(\mathrm{S})\end{array}$ & $\begin{array}{l}\mathrm{P} \\
(\mathrm{R})\end{array}$ & $\begin{array}{l}\text { Certainty } \\
\text { effect }\end{array}$ & $\begin{array}{l}\text { Mere } \\
\text { presentation }\end{array}$ & Blank \\
\hline \multirow[t]{2}{*}{1} & 1 & $4,0.8$ & 3 & 12.5 & 4 & 40 & $26^{*}$ & 10 & 10 & 72 & $-24 *$ & 49 & 73 \\
\hline & 4 & $4,0.2$ & $3,0.25$ & 19.5 & 17.5 & 66 & & 18 & 17 & 48 & & 66 & 55 \\
\hline \multirow[t]{2}{*}{2} & 7 & $4.5,0.8$ & 3 & 19.5 & 5.5 & 54 & 12 & 10 & 8.5 & 69 & $-21 *$ & 50 & 74 \\
\hline & 8 & $4.5,0.2$ & $3,0.25$ & 16 & 17 & 66 & & 17.5 & 15 & 48 & & 66 & 55 \\
\hline \multirow[t]{2}{*}{3} & 9 & $3.5,0.8$ & 3 & 20 & 4.5 & 34 & $25^{*}$ & 10 & 10 & 48 & 14 & 15 & 33 \\
\hline & 10 & $3.5,0.2$ & $3,0.25$ & 20 & 19 & 66 & & 19 & 16 & 62 & & 60 & 54 \\
\hline
\end{tabular}

$R=$ risky option, $S=$ safe option (as defined in Pairs 1,7 , and 8).

${ }^{\mathrm{a}} N(\mathrm{R})$ and $N(\mathrm{~S})$ refer to the median sample sizes for the risky and safe options, respectively.

${ }^{\mathrm{b}} P(\mathrm{R})$ refers to the proportion of respondents choosing the risky option.

${ }^{\mathrm{c}}$ The implied certainty effect in the mere-presentation and the blank conditions, respectively.

* Indicate significant differences from $0(p<0.05)$

2.3 Study 3. Can mere presentation and context attenuate the St. Petersburg paradox?

Unlike today, classical probability theory had no existence independent of its subject matter, that is, the beliefs and conduct of reasonable men (Gigerenzer et al. 1989). When the dictates of classical probability theory did not square with intuitions of reasonable men, the theorists had to return to the drawing board. The controversy over the St. Petersburg gamble epitomizes such a clash between the dictates of the theory and good sense. In this gamble, players face a scenario such as this:

The casino in St. Petersburg offers the following game: A fair coin will be flipped until it comes up heads. The number of flips will be denoted by the letter $\mathrm{k}$. The casino pays a gambler $2^{\mathrm{k}}$. What is the maximal amount of money that you are willing to pay for playing this game?

The player has a .5 chance of winning two coins, a .25 chance of winning four coins and, more generally, a $1 / 2^{n}$ chances of winning $2^{n}$ coins (where $n$ indicates the number of flips until heads comes up). The clash between good sense and probability theory arose because nobody was willing to pay substantial amounts for the right to play the gamble notwithstanding its infinite expected value (but see Jorland 1987). In order to reconcile the tension between the game's infinite expected value and gamblers' good sense (that is, their unwillingness to pay more than small sums to play the game), Daniel Bernoulli (1738) suggested replacing objective money amounts with subjective utilities. In his view, the pleasure or utility of money does not increase linearly with its monetary amount, but rather the increases in utility decline. He modeled the relation between the objective and subjective value of money in terms of a logarithmic function. In modern terminology, the resulting expected utility (EU) is defined as $E U=\sum p_{k} u\left(x_{k}\right)$, where $u\left(x_{\mathrm{i}}\right)$ is a logarithmic function defined on objective money amounts $x_{\mathrm{i}}$.

The St. Petersburg gamble offers a simple test bed for the joint impact of mere presentation and context. In its typical exposition, the gamble does not explicitly 를 Springer 
spell out the possible outcomes and their likelihoods. Thus in order to evaluate the attractiveness of the game, players have to bring, if possible, their knowledge of arithmetic and the laws of probability to the task. Had casinos taken advantage of the mere-presentation effect or the context effect, would players have been willing to pay markedly more money for the right to play the game? To answer this question, we compare the classic version of the St. Petersburg game with two variants: One variant adds explicit presentation of the possible outcomes, the second variant adds a context in which the game is compared to relatively high sure outcomes.

\subsubsection{Method}

Participants were 149 Technion students drawn from the same pool as used before. Respondents were not paid for their performance. They were randomly assigned to one of three conditions. The classic condition $(n=58)$ was modeled after the original presentation of the St. Petersburg gamble insofar as only a generic outcome was stated. Respondents read the following text:

Assume that a casino offers the following game: A fair coin will be flipped until it comes up heads. The number of flips will be denoted by the letter $\mathrm{k}$. The payoff to the player will be $2^{\mathrm{k}}$ shekels. What is the maximal amount of money that you are willing to pay in order to participate in the game?

In the chart condition $(n=53)$, the statement "The payoff to the player will be $2^{\mathrm{k}}$ shekels" was omitted. Instead, respondents were presented with the following list of outcomes and number of flips:

The payoff to the player will be:

\begin{tabular}{l}
\hline 2 shekels if $\mathrm{k}=1$ \\
4 shekels if $\mathrm{k}=2$ \\
8 shekels if $\mathrm{k}=3$ \\
16 shekels if $\mathrm{k}=4$ \\
32 shekels if $\mathrm{k}=5$ \\
64 shekels if $\mathrm{k}=6$ \\
128 shekels if $\mathrm{k}=7$ \\
256 shekels if $\mathrm{k}=8$ \\
512 shekels if $\mathrm{k}=9$ \\
1,024 shekels if $\mathrm{k}=10$ \\
2,048 shekels if $\mathrm{k}=11$ \\
4,096 shekels if $\mathrm{k}=12$ \\
8,192 shekels if $\mathrm{k}=13$ \\
16,384 shekels if $\mathrm{k}=14$ \\
32,768 shekels if $\mathrm{k}=15$ \\
65,526 shekels if $\mathrm{k}=16$ \\
131,072 shekels if $\mathrm{k}=17$ \\
262,144 shekels if $\mathrm{k}=18$ \\
524,288 shekels if $\mathrm{k}=19$ \\
$1,048,576$ shekels if $\mathrm{k}=20$ or more
\end{tabular}

Unlike in the classic condition, the game's expected value in the chart condition is no longer infinite but 21 . Irrespective of this reduction, we predicted that the explicit presentation of rare events would elicit higher willingness-to-pay (WTP) values than the classic condition. 
To investigate the impact of comparison stimuli, independent of the merepresentation effect, we also introduced the context condition $(n=38)$. Here the gamble, described generically, is compared to ten sure gains, ranging from 10 to 100 shekels:

Assume that a casino offers the following game: A fair coin will be flipped until it comes up heads. The number of flips will be denoted by the letter k. Option A promises $2^{\mathrm{k}}$ shekels. Below option $\mathrm{A}$ is compared to 10 sure gains. Please decide for each gain whether you prefer option A or the respective sure gain.

\begin{tabular}{lll}
\hline & & Sure gain \\
1 & Option A & 10 shekels with certainty \\
2 & Option A & 20 shekels with certainty \\
3 & Option A & 30 shekels with certainty \\
4 & Option A & 40 shekels with certainty \\
5 & Option A & 50 shekels with certainty \\
6 & Option A & 60 shekels with certainty \\
7 & Option A & 70 shekels with certainty \\
8 & Option A & 80 shekels with certainty \\
9 & Option A & 90 shekels with certainty \\
10 & Option A & 100 shekels with certainty \\
\hline
\end{tabular}

\subsubsection{Results}

Consistent with the mere-presentation effect, the median WTP values were more than double in the chart condition than in the classic condition-10 versus 4.5 $(\chi 2(1)=9.29, p<.005$, using the Brown-Mood median test). In addition, the WTP values of about a third of respondents - 32\% (17 out of 53) - in the chart condition exceeded the game's expected value of 21 versus $19 \%$ (11 of the 58 ) in the classic condition. Thus, merely presenting the rare yet lucrative outcomes increased their inferred impact and made a third of people behave as if they were risk seeking.

Consistent with the hypothesis that the context of comparison stimuli affects choices and the inferred impact of rare events, the percentage of people preferring the gamble over 10 shekels was $58 \%$ (22 out of 38 ) in the context condition, whereas it was only $22 \%$ (13 out of 58 ) in the classic condition. Similarly, $42 \%$ (16 out of 38 ) of respondents in the context condition preferred the gamble over 20 shekels, whereas only $19 \%$ (11 out of 58$)$ did so in the classic condition. These differences are significant $(\mathrm{t}(94)=3.52, p<.0001$ and $\mathrm{t}(94)=2.52, p<.05$, respectively). By comparing the gamble to a distribution of comparison stimuli consisting of sure gains, the gamble was perceived to be more attractive. Thus, people behaved as if rare lucrative events in the St. Petersburg gamble received more weight than in the classic condition.

On one interpretation of choices in the chart condition, people - in the process of translating number of flips into respective probabilities - may overestimate the probabilities of rare events. This overestimation could then drive their favorable 
impression of the gamble, relative to the classic condition. To address this possibility we ran a variant of the chart condition in which participants were first asked to estimate the probabilities of the 20 outcomes of the gamble, and then to make their evaluation. Specifically, participants $(n=35)$ were asked to write their probability estimate next to each of the listed outcomes (in an empty column that was added to the aforementioned table). Four participants correctly estimated the probability of all 20 outcomes. Twenty-seven participants erred in the direction of underestimation. Specifically, 26 of them estimated the probability of the first 19 outcomes correctly, but incorrectly estimated the probability of the 20th outcome as 1/1,048,576 (the objective probability is $2 / 1,048,576$ ). This mistake reduced the implied expected value from 21 to 20 . Only 4 of the 35 participants overestimated the respective probabilities. Two of these errors reflected a lapse in attention (i.e., jumping a line) and the remaining two involved an error in the computation of the probability of the 20th outcome. People's median bid was 10 shekels, a value identical to the median bid in the chart condition. Finally, eliminating the four participants who overestimated the relevant probabilities did not affect the median bid. From these findings we conclude that the higher WTP values in the chart condition, relative to the classic condition, are not simply due to overestimation of the likelihood of rare events.

Taken together, the findings in Study 3 reflect that both the mere presentation of rare events as well as the design of comparison stimuli can have a substantial effect on people's choices, and by extension, on the implied weighting of rare events.

2.4 Study 4. Can mere presentation and context reverse the St. Petersburg paradox?

Study 3 suggests that mere presentation of rare events and the requirement to consider a context of alternatives reduce the implied risk aversion in variants of the St. Petersburg problem. We now examine whether people can be turned from risk averse to risk seeking gamblers in the St. Petersburg problem.

\subsubsection{Method}

Participants were 20 Technion students. They responded to a short questionnaire, and were not paid for their performance. Respondents read the following instructions:

Assume that a casino offers the following game: A fair coin will be flipped until it comes up heads. The number of flips will be denoted by the letter k. Option A promises the following payoff:

\footnotetext{
2 shekels if $\mathrm{k}=1$

4 shekels if $\mathrm{k}=2$

8 shekels if $\mathrm{k}=3$

16 shekels if $\mathrm{k}=4$

32 shekels if $k \geq 5$
} 
Below option A is compared to 15 sure gains. Please decide for each gain whether you prefer option A or the respective sure gain.

\begin{tabular}{lll}
\hline & & Sure gain \\
1 & Option A & 3 shekels with certainty \\
2 & Option A & 5 shekels with certainty \\
3 & Option A & 7 shekels with certainty \\
4 & Option A & 9 shekels with certainty \\
5 & Option A & 11 shekels with certainty \\
6 & Option A & 13 shekels with certainty \\
7 & Option A & 15 shekels with certainty \\
8 & Option A & 17 shekels with certainty \\
9 & Option A & 19 shekels with certainty \\
10 & Option A & 21 shekels with certainty \\
11 & Option A & 23 shekels with certainty \\
12 & Option A & 25 shekels with certainty \\
13 & Option A & 27 shekels with certainty \\
14 & Option A & 29 shekels with certainty \\
15 & Option A & 31 shekels with certainty \\
\hline
\end{tabular}

\subsection{Results}

The expected value of this variant of the St. Petersburg gamble (i.e., option $A$ ) amounts to 6 shekels. Notwithstanding the gamble's expected value, 18 of $20(90 \%)$ respondents preferred it over 7 shekels. This proportion is significantly larger than $50 \%$ (sign test, $p<.01$ ). Thus, in this version of the St. Petersburg game, most respondents proved to be risk seeking. The median certainty equivalent was 10 shekels - a value that proved to be significantly larger than the corresponding value in the classic condition of Study $3(\mid 2(1)=17.6, p<.0001$, using the Brown-Mood median test). By taking advantage of the mere-presentation and the context effects, one can turn otherwise risk-averse respondents into pronounced risk seekers in the St. Petersburg gamble.

\section{General discussion}

Kahneman and Tversky (1979) suggested that people deviate from optimal weighting of rare events in two possible directions, overweighting and neglect. In the context of decisions from incomplete descriptions, we investigated two possible factors that determine the implied direction of the deviation. Both factors - the effects of comparison stimuli (context) and of mere presentation-are well-known beyond research on risky choice. Study 1 showed how exquisitely sensitive estimated certainty equivalents are to the context, that is, the list of alternatives to which the prospect is compared. When most comparison alternatives were similar to the rare event (i.e., the context is skewed toward the rare event such that the rare events and most comparison stimuli are located on the same side of the prospect's expected value) the implied certainty equivalents suggested overweighting of rare events. When most alternatives were similar to the most likely outcome, however, certainty equivalents suggested that rare events have less impact than they deserve. 
In other words, certainty equivalents, and conclusions regarding the impact of rare events based on certainty equivalents, are strongly affected by the comparison stimuli.

Using the certainty effect, Study 2 showed that when rare events were explicitly presented, respondents made choices as if rare events had more impact than they deserved, relative to their objective probability. When rare events were not explicitly presented and people had to rely on their memory of the encountered outcomes, choices were made as if rare events had less impact than they deserved. A similar result was observed in Study 3. Using the classic St. Petersburg gamble, we demonstrated that people's willingness-to-pay price more than doubled when the desirable rare events were explicitly presented, relative to a generic description of the gamble. Finally, in Study 4, we showed that by combining the effects of context and mere presentation, almost every respondent could be turned into a risk seeker in the St. Petersburg gamble. In what follows, we describe a quantitative model of the joint implication of both factors.

\subsection{Two critiques and a simple model}

The value of the current analysis can be questioned on two main grounds. The first involves the observation that the two well-known factors studied here are, indeed, well known. Therefore, the current results do not reveal anything new. The second critique is based on the assertion that there may be good reasons why the two wellknown factors, considered here, are ignored by leading descriptive models of choice behavior. According to this critique, the effects of these factors are likely to be weak and, perhaps, best conceptualized as noise. We believe that the second critique implies a good answer to the first critique. The observation that leading decisionmaking models ignore the context and mere-presentation effects suggests that our understanding of these effects is limited.

The main goal of the current section is to respond to the second critique. The basic idea behind our answer is the observation that the leading models that ignore the context and mere-presentation effects focus on relatively narrow experimental paradigms in which these effects imply overweighting of rare events. For example, in all the conditions considered by Tversky and Kahneman (1992) and in Fox and Tversky (1998) the possible outcomes were presented before the participants. ${ }^{5}$ It is easy to see that in these environments the abstraction of context and merepresentation effects can be replaced with the assumption of uniform overweighting of rare events. We conjecture that the explicit abstraction of the context and merepresentation effects is likely to be necessary if the goal is to address a wider set of situations with a general model. The following section evaluates this conjecture by considering a simple model of decisions from experience.

\subsubsection{The primed-sampler model}

The current analysis starts with the value-updating model proposed by Hertwig, Barron, Weber, and Erev (2006) to account for decisions from experience. Its basic

\footnotetext{
${ }^{5}$ As noted by Blavatskyy (2005), influential models, like cumulative prospect theory, cannot capture behavior in the Allais and St. Petersburg problems with the same set of parameters.
} 
idea is the observation that reliance on small samples of experiences (see related assumptions in Kareev 2000; Fiedler 2000; Osborne and Rubinstein 1998) implies underweighting of rare events. The reason is that rare events tend to be underrepresented in small samples (see Hertwig et al. 2006 for details). The model provides a useful summary of the results of the experience condition summarized in Table 1 (and of the blank condition in Study 2), but it fails to capture the results of Study 1 and the mere-presentation condition in Study 2. To accommodate these results, we extend the value-updating model. The most important extension involves the distinction between two sampling processes: Sampling from the objective payoff distributions that results in "experiences" or memory instances (see Logan 1988), and covert sampling from these experiences. The "primed-sampler" model assumes that the covert sampling process determines choice behavior, and is sensitive to priming in terms of context and mere presentation. Specifically, context and mere presentation determine the implied weighting of rare events because they prime particular experiences. The model can be summarized with the following assumptions:

Sampling from the objective distributions The decision maker is assumed to draw $\eta$ samples (with replacement) from the objective payoff distributions. We refer to the outcomes of this sampling process as "experiences." Notice that in Studies 1 and 2 this sampling is observable. The mean value of $\eta$ over the two studies is around 20.

Covert sampling from experiences In order to evaluate the different alternatives the decision maker is assumed to draw $\kappa(\kappa<\eta)$ samples (with replacement) from the stored experiences of each alternative. This process is assumed to be sensitive to two potential sources of priming: the presentation of the possible outcomes, and the presentation of the comparison stimuli. The exact effect of these factors is captured with the assumption that each of the $\kappa$ covert samples is drawn using a sampling algorithm that can be in one of three mutually exclusive priming states:

The first state involves "no priming," and entails a random draw from the $\eta$ experiences. This state occurs when the decision maker is not primed (i.e., no presentation of the outcomes and comparison stimuli as in the blank condition of Study 2), or when the decision maker ignores the priming (the probability that the priming is disregarded, denoted by $\alpha$, is a free parameter). The second state involves "priming by presentation". Under this state, one of the presented outcomes is randomly selected, and the experience most similar to this outcome is drawn. For example, consider the evaluation of the prospect " 4 with probability .8; 0 otherwise." First, one of the two outcomes ( 0 or 4$)$ is randomly selected. Then, from the stored experiences the one most similar to the selected one is drawn. This kind of priming occurs with probability $1-\alpha$ when the outcomes are the only source of priming (as in the mere presentation condition in study 2$)$, and with probability $(1-\alpha) \beta$ when both priming factors are active. Finally, the third state involves "priming by context" according to which one of the presented comparison stimuli is randomly selected, and the experience (among the stored experiences) most similar to this stimulus is drawn. It is interesting to note that the impact of priming with the covert sampling process implies a bias toward equal weighting of the different outcomes. Viscusi (1989) shows that such a bias can explain the main behavioral deviations from expected utility theory, and can be a product of Bayesian reasoning. 
Updating rule Each of the $\kappa$ drawn experiences enters the calculation of the value of the gambles with the same weight. In order to calculate a gamble's "average subjective value" the sampled experiences are summed, separately for each gamble, and then divided by sample size.

Choice rule The decision maker selects the gamble with the highest "average subjective value." If both gambles' values are equal, the tie-breaking rule depends on the available information. It assumes random choice when the possible outcomes are not presented, and a "priority-tie-breaking rule" (see Brandstätter, Gigerenzer, and Hertwig 2006) otherwise. According to this rule the first tie-breaking criterion is the minimum outcome, the second criterion is the maximum outcome, and a random choice is assumed if neither criterion breaks the tie. ${ }^{6}$

The model has four parameters: The number of experiences $(\eta)$, the sample size from the experiences $(\kappa)$, the probability of considering the presented outcomes or the context $(\alpha)$, and the relative attention given to the outcomes when the alternatives and the outcomes are presented $(\beta)$. In order to reduce the number of free parameters we set the value of $\eta$ to equal 20 - an approximation of the mean value observed in the studies. The other parameters were estimated to fit the data. To determine whether the primed-sampler model can describe the main results observed in Studies 1 and 2, we simulated virtual decision makers. They arrived at their choices on the basis of the model's policy. We then compared the choice proportions predicted by the model to the empirically observed choice proportions. The simulation proceeded as follows:

1. A sample of 20 experiences was drawn from the objective distributions, and then a sample of $\kappa$ "drawn experiences" was selected using the algorithm described above.

2. The average values in the samples of "selected experiences" were computed.

3. The gamble with the highest value was selected (and the tie-breaking rule was used in case of a tie).

Tables 1, 2, and 3 present the model's predictions with the best-fitting parameters of the 30 observed choice proportions (i.e., six times three proportions in Table 1 and six times two proportions in Table 3). The estimated parameters are $\kappa=5, \alpha=.65$, and $\beta=.05$. The correlation between predicted and observed proportions is .86 ; the mean square deviation between the observed and predicted proportions is .011 . In addition to the good quantitative fit, the model reproduces the main qualitative results. In particular, it captures the Allais paradox in the AS-Rare context (Study 1) and the mere-presentation condition (Study 2), as well as the reversed Allais pattern in the experience condition (Hertwig et al. 2004), the AS-Likely context (Study 1), and the blank condition (Study 2).

In order to reduce the risk of overfitting, we also conducted a "leave one out" analysis. First, the model's parameters were estimated based on all the 30 distinct

\footnotetext{
${ }^{6}$ This rule is similar but not identical to the priority rule in the priority heuristic (Brandstätter et al. 2006). According to this rule, reasons are considered in the order: minimum gain, probability of minimum gain and maximum gain. Because there are no explicit probabilities available, the priority-tie-breaking rule skips the second reason.
} 
subsets of 29 observations (each subset included all but one of the 30 choice proportions considered above). Second, we used each of the 30 estimated parameter sets to predict the left-out observation. The results revealed only small differences between the leave-one-out analysis and the original analysis: The estimated value of $\kappa$ stayed at 5 in all 30 subsets. The value of $\alpha$ was between 0.7 and 0.55 , and the value of $\beta$ was below 0.21 in all cases. The MSD and correlation scores were 0.014 and 0.83 , respectively.

An additional indication for the relative robustness of the results is provided by the evaluation of the model's prediction of the certainty equivalent values. With the parameters estimated above the correlation between the observed and predicted mean certainty equivalent values is .995 (see Table 2) and the mean squared deviation is 0.17 . Thus, the model also captures the large effect of the range of certain payoffs on the estimated certainty equivalents. Of course, there are other and possibly better ways in which the context and mere-presentation effects can be captured. The important point, however, is that integrating these factors into the descriptive models of choice is likely to increase their explanatory power.

\subsubsection{Sampling without direct experience}

The primed-sampler model was designed to address situations in which the decision maker can rely on previous experiences when considering the different alternatives. What about situations such as the different variants of the St. Petersburg paradox in which the decision maker cannot access objective experience? According to one extension of the model, objective experience is replaced with an unbiased simulation of $\eta$ (with $\eta>\kappa$ ) draws from the prospect's payoff distribution. Can this extension of the model reproduce the results of Studies 3 and 4? Using the parameter values estimated above $(\kappa=5, \alpha=.65$, and $\beta=.05)$, we examined different values of $\eta$. The results reveal that with $\eta=15$ the model reproduces the main results: It implies strong risk aversion in the classical St. Petersburg problem (median WTP is 4.8), weaker risk aversion in both chart and context conditions of Study 3 (median WTP of 11.6 and median certainty equivalents of 20, respectively), and risk-seeking behavior in Study 4 (median certainty equivalence of 8).

\subsection{Context, mere presentation and adaptive behavior}

Abstracting the context and mere-presentation effects in terms of the primed-sampler model illustrates the fact that both can lead to systematic deviations from optimal choice. Can learning or evolution reduce their impact? Before answering this question, let us first emphasize our belief that in principle sensitivity to context and mere presentation is adaptive and socially reasonable in light of norms of communication (e.g., Grice 1989). Considering context as informative can be adaptive to the extent that options tend to be compared to similarly attractive (rather than dissimilar) alternatives. Thus, the attractiveness of the comparison stimuli can be a good approximation of the attractiveness of the target option. Relatedly, there are reasons for a speaker to frame a situation in terms of rare rather than likely events, and for a speaker to find this framing particularly informative. For example, 
McKenzie, Ferreira, Mikkelsen, McDermott, and Skrable (2001) found that people have a tendency, perhaps even a strong tendency, to phrase conditional hypotheses in terms of rare events. In so doing, their behavior reflects the fact that when testing hypotheses, rare or unexpected observations are normatively more informative than common observation.

Notwithstanding these regularities, there are situations in which reliance on context and mere presentation can be become disadvantageous. For illustration, consider the world of casinos. In order to attract gamblers, casinos use the merepresentation effect through eye-catching presentations of high but unlikely prizes. In fact, if casinos offered the St. Petersburg problem, Study 3 shows how they would be able to boost players' willingness to pay more money for the right to play the gamble. Effective learning should reduce mere-presentation effects in such a setting. However, as Haruvy, Erev, and Sonsino (2001) have noted: When the payoff variability is large (another common property of the gambles offered in casinos) learning is not likely to be effective.

\subsection{Concluding remarks}

Two well-known psychological factors whose impact has been described in many areas of human cognition and perception also affect risky choice, certainty equivalents and willingness-to-pay values. These behavioral facts are worthy of consideration independent of whether risky choice is modeled in terms of non-linear probability weighting functions (e.g., cumulative prospect theory; Tversky and Kahneman 1992) or in terms of heuristics that treat probabilities linearly (e.g., priority heuristic; Brandstätter et al. 2006). As we have shown, by taking advantage of the mere-presentation effect and the context effect one can design decision environments that yield behavior that-when interpreted in terms of non-linear probability weighting functions - implies either overweighting or underweighting of rare events. The mere-presentation effect and the context effect are thus two entries in a list of environmental factors that determine people's choices, and, by extension, researchers' interpretation of the underlying processes.

The context effect is of particular importance in the elicitation and interpretation of certainty equivalents. The mere-presentation effect is likely to be of relevance in explaining the disparity between decisions from description and decisions from incomplete description. In the former, all outcomes and their probability are fully displayed in front of the respondent, regardless of their rarity or ubiquity. As Hertwig et al. (2006, p. 88) suggested, "the propositional representations of gambles in decisions from description-for instance, '32 with probability .1; 0 otherwise' - put more equal emphasis on the two possible outcomes than the discrepancy between their actual probabilities' occurrences warrants. If attention translates into decision weights as some research suggests (Weber and Kirsner 1996), then, other things being equal, the psychological weight of rare and common events will indeed be closer to one another then they should be." In decisions from incomplete description, in contrast, the absence or just the underrepresentation of rare events (due to small samples; Hertwig et al. 2004) may amplify the difference in the psychological weight of rare and common events. 
To conclude, really good things and really bad things happen rarely. Most of us experience only one true love. Very few of us hit the jackpot. Few of us are lucky enough to graduate from Harvard or Yale, and become U.S. Senators, CEOs, or movie stars. By the same token, however, few of us - fortunately-become victims of an airplane crash, are drafted during time of war, or are born with a debilitating birth defect. Events representing our brightest dreams and our darkest nightmares occur infrequently. Mundane events, in contrast, are just that-everyday events. Captivating our imagination, just what impact do rare events have on our decisions? The present studies have demonstrated that the implied psychological impact of rare events is not predestined but depends on two robust factors, context and mere presentation. We are confident that other factors will be added to the list of determinants.

\section{References}

Allais, Maurice. (1953). "Le Comportement de l'Homme Rationnel devant le Risque: Critique des Postulats et Axiomes de l'Ecole Américaine," Econometrica 21, 503-546.

Barron, Gregory M. and Ido Erev. (2003). "Small Feedback-Based Decisions and Their Limited Correspondence to Description-Based Decisions," Journal of Behavioral Decision Making 16, 215-233.

Bernoulli, Daniel. (1738). "Exposition of a New Theory on the Measurement of Risk," Comentarii Academiae Scientiarum Imperialis Petropolitanae. Translated and reprinted in Econometrica 22, 23-36, (1954).

Birnbaum, Michael H. (1992). "Violations of Monotonicity and Contextual Effects in Choice-Based Certainty Equivalents," Psychological Science 3, 310-314.

Birnbaum, Michael H. and Teresa Martin. (2003). "Generalization across People, Procedures, and Predictions: Violations of Stochastic Dominance and Coalescing." In Sandra L. Schneider and James Shanteau (eds), Emerging Perspectives on Decision Research. New York: Cambridge University Press.

Blavatskyy, Pavlo. (2005). "Back to the St. Petersburg Paradox?" Management Science 51, 677-678.

Brandstätter, Eduard, Gerd Gigerenzer, and Ralph Hertwig. (2006). "The Priority Heuristic: Making Choices Without Trade-offs," Psychological Review 113, 409-432.

Chateauneuf, Alain and Peter Wakker. (1999). "An Axiomatization of Cumulative Prospect Theory for Decision Under Risk," Journal of Risk and Uncertainty 18(2), 137-145.

Erev, Ido, Thomas S. Wallsten, and David V. Budescu. (1994). "Simultaneous Over- and Underconfidence: The Role of Error in Judgment Processes," Psychological Review 101, 519-527.

Erev, Ido, Dvorit Shimonowitch, Amos Schurr, and Ralph Hertwig. (2008). "Base Rates: How to Make the Intuitive Mind Appreciate or Neglect Them." In Henning Plessner, Cornelia Betsch and Tilmann Betsch (eds), Intuition in Judgment and Decision Making. New York: Lawrence Erlbaum.

Fiedler, Klaus. (2000). "Beware of Samples! A Cognitive-Ecological Sampling Approach to Judgment Biases," Psychological Review 107, 659-676.

Fiedler, Klaus, Thomas Armbruster, Stefanie Nickel, Eva Walther, and Judith Asbeck. (1996). "Constructive Biases in Social Judgment: Experiments on the Self-Verification of Question Contents," Journal of Personality and Social Psychology 71, 861-873.

Fox, Craig R. and Amos Tversky. (1998). "A Belief-Based Account of Decision Under Uncertainty," Management Science 44, 879-895.

Garner, Wendell R. (1954). "Context Effects and the Validity of Loudness Scales," Journal of Experimental Psychology 48, 218-224.

Gigerenzer, Gerd, Zeno Swijtink, Theodore Porter, Lorraine Daston, John Beatty, and Lorenz Kriiger. (1989). The Empire of Chance. How Probability Changed Science and Everyday Life. Cambridge, UK: Cambridge University Press.

Gregory, Larry W., Robert B. Cialdini, and Kathleen M. Carpenter. (1982). "Self-Relevant Scenarios as Mediators of Likelihood Estimates and Compliance: Does Imagining Make it So?" Journal of Personality and Social Psychology 43, 89-99. 
Gregory, Robin, Sarah Lichtenstein, and Paul Slovic. (1993). "Valuing Environmental Resources: A Constructive Approach," Journal of Risk and Uncertainty 7, 177-197.

Grice, Herbert P. (1989). Studies in the Way of Words. Cambridge, MA: Harvard University Press.

Hasher, Lynn, David Goldstein, and Thomas Toppino. (1977). "Frequency and the Conference of Referential Validity," Journal of Verbal Learning and Verbal Behavior 16, 107-112.

Harbaugh, William T., Kate Krause, and Lise Vesterlund. (2004). "Prospect Theory in Choice and Pricing Tasks," EconPapers (No. 2002-2), University of Oregon Economics Department Working Papers, available at: http://econpapers.repec.org/paper/oreuoecwp/2002-02.htm.

Hertwig, Ralph, Gregory M. Barron, Elke Weber, and Ido Erev. (2004). "Decisions from Experience and the Effect of Rare Events in Risky Choice," Psychological Science 15, 534-539.

Hertwig, Ralph, Gregory M. Barron, Elke Weber, and Ido Erev. (2006). "The Role of Information Sampling in Risky Choice." In Klaus Fiedler and Peter Juslin (eds), Information Sampling and Adaptive Cognition. Cambridge, UK: Cambridge University Press.

Hertwig, Ralph, Gerd Gigerenzer, and Ulrich Hoffrage. (1997). "The Reiteration Effect in Hindsight Bias," Psychological Review 104, 194-202.

Haruvy, Ernan, Ido Erev, and Doron Sonsino. (2001). "The Medium Prizes Paradox: Evidence from a Simulated Casino," Journal of Risk and Uncertainty 22, 251-261.

Ish Shalom, Taly. (2004). "Rats, Humans, and the Common Ratio Effect." M.Sc. Thesis, Haifa, Israel: Technion Israel Institute of Technology.

Jorland, Gérard. (1987). "The Saint Petersburg Paradox 1713-1937." In Lorenz Krüger, Lorraine J. Daston, and Michael Heidelberger (eds), The Probabilistic Revolution Vol. I: Ideas in History. Cambridge, MA: MIT Press.

Kahneman, Daniel and Amos Tversky. (1979). "Prospect Theory: An Analysis of Decision under Risk," Econometrica 47, 263-291.

Kareev, Yaakov. (2000). "Seven (Indeed, Plus or Minus Two) and the Detection of Correlations," Psychological Review 107, 397-402.

Logan, Gordon D. (1988). “Toward an Instance Theory of Automatization,” Psychological Review 95, $492-527$.

Luce, R. Duncan and Howard Raiffa. (1957). Games and Decisions. New York: Wiley.

McKenzie, Craig R.M., Victor S. Ferreira, Laurie A. Mikkelsen, Kristine J. McDermott, and Ryan P. Skrable. (2001). "Do Conditional Hypotheses Target Rare Events?" Organizational Behavior and Human Decision Processes 85, 291-309.

Osborne, Martin J. and Ariel Rubinstein. (1998). "Games with Procedurally Rational Players," American Economic Review 88, 834-847.

Prelec, Drazen. (2000). "Compound Invariant Weighting Functions in Prospect Theory.” In Daniel Kahneman and Amos Tversky (eds), Choices, Values, and Frames. New York: Cambridge University Press.

Stewart, Neil, Nick Chater, and Gordon D.A. Brown. (2006). "Decision by Sampling," Cognitive Psychology 53, 1-26.

Tversky, Amos and Daniel Kahneman. (1992). "Advances in Prospect Theory: Cumulative Representation of Uncertainty," Journal of Risk and Uncertainty 9, 195-230.

Tversky, Amos and Derek J. Koehler. (1994). "Support Theory: A Nonextensional Representation of Subjective Probability," Psychological Review 101, 547-567.

Viscusi, W. Kip. (1989). "Prospective Reference Theory: Toward an Explanation of the Paradoxes," Journal of Risk and Uncertainty 2, 235-264.

Wakker, Peter P. and Amos Tversky. (1993). "An Axiomatization of Cumulative Prospect Theory," Journal of Risk and Uncertainty 7, 147-175.

Weber, Elke U. and Britt Kirsner. (1996). "Reasons for Rank-dependent Utility Evaluation," Journal of Risk and Uncertainty 14, 41-61.

Weber, Elke U., Sharoni Shafir, and Ann-Renée Blais. (2004). "Predicting Risk Sensitivity in Humans and Lower Animals: Risk as Variance or Coefficient of Variation," Psychological Review 111, 430-445.

Wu, George and Richard Gonzalez. (1998). "Common Consequence Conditions in Decision Making under Risk," Journal of Risk and Uncertainty 16, 115-139.

Wu, George and Richard Gonzalez. (1999). "Nonlinear Decision Weights in Choice Under Uncertainty," Management Science 45, 74-85. 